\title{
Inertia of Loewner Matrices
}

\author{
Rajendra Bhatia ${ }^{1}$, Shmuel Friedland ${ }^{2}$, Tanvi Jain ${ }^{3}$ \\ ${ }^{1}$ Indian Statistical Institute, New Delhi 110016, India \\ rbh@isid.ac.in \\ ${ }^{2}$ Department of Mathematics, Statistics and Computer Science, University of \\ Illinois at Chicago, Chicago, 60607-7045, USA \\ friedlan@uic.edu \\ ${ }^{3}$ Indian Statistical Institute, New Delhi 110016, India \\ tanvi@isid.ac.in
}

\begin{abstract}
Given positive numbers $p_{1}<p_{2}<\cdots<p_{n}$, and a real number $r$ let $L_{r}$ be the $n \times n$ matrix with its $i, j$ entry equal to $\left(p_{i}^{r}-p_{j}^{r}\right) /\left(p_{i}-p_{j}\right)$. A well-known theorem of C. Loewner says that $L_{r}$ is positive definite when $0<r<1$. In contrast, R. Bhatia and J. Holbrook, (Indiana Univ. Math. J, 49 (2000) 1153-1173) showed that when $1<r<2$, the matrix $L_{r}$ has only one positive eigenvalue, and made a conjecture about the signatures of eigenvalues of $L_{r}$ for other $r$. That conjecture is proved in this paper.
\end{abstract}

AMS Subject Classifications : 15A48, 47B34.

Keywords : Loewner Matrix, inertia, positive definite matrix, conditionally positive definite matrix, Sylvester's law, Vandermonde matrix.

\section{Introduction}

Let $f$ be a real-valued $C^{1}$ function on $(0, \infty)$. Let $p_{1}<p_{2}<\cdots<p_{n}$ be any $n$ points in $(0, \infty)$. The $n \times n$ matrix

$$
L_{f}\left(p_{1}, \ldots, p_{n}\right)=\left[\frac{f\left(p_{i}\right)-f\left(p_{j}\right)}{p_{i}-p_{j}}\right]_{i, j=1}^{n}
$$

is called a Loewner matrix associated with $f$. It is understood that when $i=j$, the quotient in (1) represents the limiting value $f^{\prime}\left(p_{i}\right)$. Of particular interest to us are the functions $f(t)=t^{r}, r \in \mathbb{R}$. In this case we write $L_{r}$ for $L_{f}\left(p_{1}, \ldots, p_{n}\right)$, where the roles of $n$ and $p_{1}, \ldots p_{n}$ can be inferred from the context. Thus $L_{r}$ is the $n \times n$ matrix

$$
L_{r}=\left[\frac{p_{i}^{r}-p_{j}^{r}}{p_{i}-p_{j}}\right]_{i, j=1}^{n} .
$$

Loewner matrices are important in several contexts, of which we mention two that led to the present study. (The reader may see Section 4.1 of [12] for an 
excellent discussion of both these aspects of Loewner matrices.) The function $f$ on $(0, \infty)$ induces, via the usual functional calculus, a matrix function $f(A)$ on the space of positive definite matrices. Let $D f(A)$ be the Fréchet derivative of this function. This is a linear map on the space of Hermitian matrices. The Daleckii-Krein formula describes the action of this map in terms of Loewner matrices. Choose an orthonormal basis in which $A=\operatorname{diag}\left(p_{1}, \ldots, p_{n}\right)$. Then the formula says that for every Hermitian $X$

$$
D f(A)(X)=L_{f}\left(p_{1}, \ldots, p_{n}\right) \circ X,
$$

where $A \circ B$ stands for the entrywise product $\left[a_{i j} b_{i j}\right]$ of $A$ and $B$.

The function $f$ is said to be operator monotone on $(0, \infty)$ if $A \geq B>0$ implies $f(A) \geq f(B)$. (As usual $A \geq 0$ means $A$ is positive semidefinite.) A fundamental theorem due to Charles Loewner says that $f$ is operator monotone if and only if all Loewner matrices associated with $f$ (for every $n$ and for every choice $\left.p_{1}, \ldots, p_{n}\right)$ are positive semidefinite. Another basic fact, again proved first by Loewner, says that $f(t)=t^{r}$ is operator monotone if and only if $0 \leq r \leq 1$. See 1] Chapter V.

Combining these various facts with some well-kown theorems on positive linear maps 2] one can see that if $f$ is operator monotone, then the norm of $D f(A)$ obeys the relations

$$
\|D f(A)\|=\|D f(A)(I)\|=\left\|f^{\prime}(A)\right\|,
$$

and is therefore readily computable. In particular, for the function $f(t)=t^{r}$ if we write $D A^{r}$ for $D f(A)$, then (4) gives

$$
\left\|D A^{r}\right\|=\left\|r A^{r-1}\right\|, \quad \text { for } 0 \leq r \leq 1 .
$$

This was first noted in 3, and used to derive perturbation bounds for the operator absolute value. Then in 8 Bhatia and Sinha showed that the relation (5) holds also for $-\infty<r<0$ and for $2 \leq r<\infty$ but, mysteriously, not for $1<r<\sqrt{2}$. The case $\sqrt{2} \leq r<2$, left open in this paper, was resolved in [4] by Bhatia and Holbrook, who showed that here again the relation (5) is valid.

One ingredient of the proof in [4] is their Proposition 2.1 which says that when $1<r<2$, the $n \times n$ matrix $L_{r}$ has just one positive eigenvalue. We have remarked earlier that when $0<r<1$, the matrix $L_{r}$ is positive semidefinite and therefore, none of its eigenvalues is negative. This contrast as $r$ moves from $(0,1)$ to $(1,2)$ is intriguing, and raises the natural question about the behaviour of eigenvalues of $L_{r}$ for other values of $r$. Bhatia and Holbrook [4] made a conjecture about this behaviour and established a small part of it: they settled the cases $r=1,2, \ldots, n-1$ apart from $0<r<1$ and $1<r<2$ already mentioned. The main goal of this paper is to prove this conjecture in full. This is our Theorem 1.1.

Let $A$ be an $n \times n$ Hermitian matrix. The inertia of $A$ is the triple

$$
\operatorname{In}(A)=(\pi(A), \zeta(A), \nu(A)),
$$


where $\pi(A)$ is the number of positive eigenvalues of $A, \zeta(A)$ is the number of zero eigenvalues of $A$, and $\nu(A)$ the number of negative eigenvalues of $A$. Theorem 1.1 describes the inertia of $L_{r}$ as $r$ varies over $\mathbb{R}$. It is easy to see that the inertia of $L_{-r}$ is the opposite of the inertia of $L_{r}$; i.e. $\pi\left(L_{-r}\right)=\nu\left(L_{r}\right)$ and $\nu\left(L_{-r}\right)=\pi\left(L_{r}\right)$. So we confine ourselves to the case $r>0$.

Theorem 1.1. Let $p_{1}<p_{2}<\cdots<p_{n}$ and $r$ be any positive real numbers and let $L_{r}$ be the matrix defined in (2). Then

(i) $L_{r}$ is singular if and only if $r=1,2, \ldots, n-1$.

(ii) At the points $r=1,2, \ldots, n$, the inertia of $L_{r}$ is given as follows:

$$
r=2 k \Rightarrow \operatorname{In}\left(L_{r}\right)=(k, n-r, k),
$$

and

$$
r=2 k-1 \Rightarrow \operatorname{In}\left(L_{r}\right)=(k, n-r, k-1) .
$$

(iii) If $0<r<n$ and $r$ is not an integer, then

$$
\lfloor r\rfloor=2 k \Rightarrow \operatorname{In}\left(L_{r}\right)=(n-k, 0, k)
$$

and

$$
\lfloor r\rfloor=2 k-1 \Rightarrow \operatorname{In}\left(L_{r}\right)=(k, 0, n-k) .
$$

(iv) If $r>n-1$, then $\operatorname{In}\left(L_{r}\right)=\operatorname{In}\left(L_{n}\right)$.

(v) Every nonzero eigenvalue of $L_{r}$ is simple.

It is helpful to illustrate the theorem by a picture. Figure 1 is a diagram of the (scaled) eigenvalues of a $6 \times 6$ matrix $L_{r}$ when $p_{i}$ are fixed and $r$ varies. Some of the eigenvalues are very close to zero. To be able to distinguish between them the vertical scale has been expanded.

We have already mentioned that for $0<r<1$, statement (iii) of Theorem 1.1 follows from Loewner's theorem, and for $1<r<2$ it was established in 4 . The case $2<r<3$ was accomplished by Bhatia and Sano in [7]. We briefly explain this work.

Let $\mathcal{H}_{1}$ be the space

$$
\mathcal{H}_{1}=\left\{x=\left(x_{1}, \ldots, x_{n}\right): \sum_{i=1}^{n} x_{i}=0\right\} .
$$

An $n \times n$ Hermitian matrix $A$ is said to be conditionally positive definite if $\langle x, A x\rangle \geq 0$ for all $x \in \mathcal{H}_{1}$, and if $-A$ has this property, then we say that $A$ is conditionally negative definite. Since $\operatorname{dim} \mathcal{H}_{1}=n-1$, a nonsingular conditionally positive definite matrix which is not positive definite has inertia $(n-1,0,1)$. 
Eigenvalues of $L_{r} ; n=6,0 \leq r \leq 10$

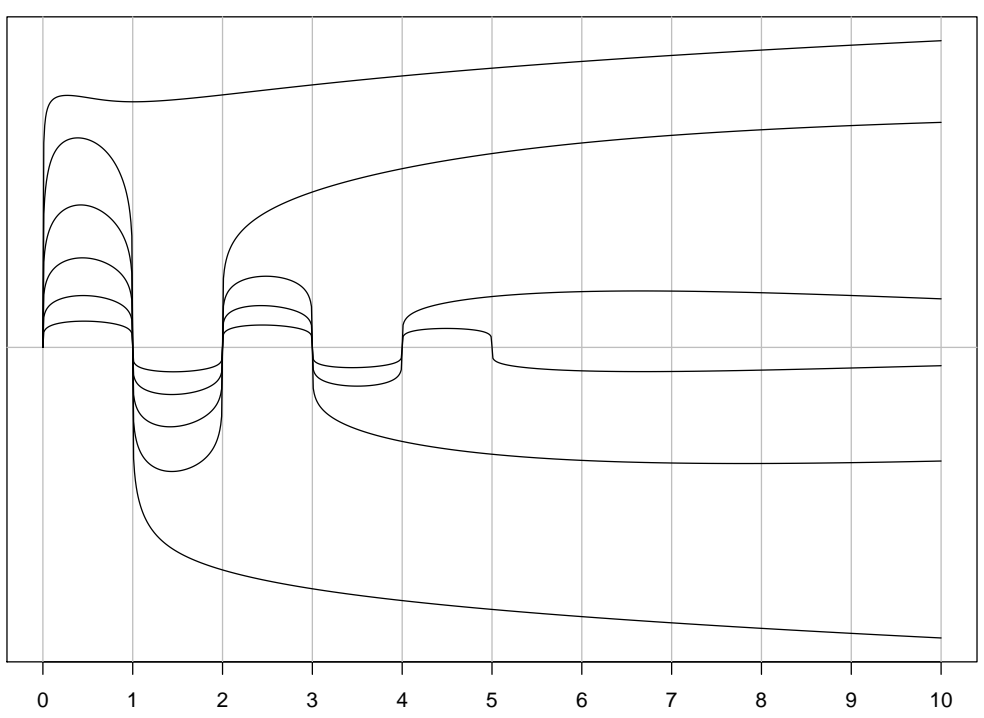

Figure 1:

In [7] it was shown that when $1<r<2$, the matrix $L_{r}$ is nonsingular and conditionally negative definite. It follows that $\operatorname{In}\left(L_{r}\right)=(1,0, n-1)$, a fact established earlier in [4]. It was also shown in [7] that when $2<r<3$, the matrix $L_{r}$ is nonsingular and conditionally positive definite. From this it follows that $\operatorname{In}\left(L_{r}\right)=(n-1,0,1)$.

More generally, Bhatia and Sano [7] showed that $f$ on $(0, \infty)$ is operator convex if and only if all Loewner matrices $L_{f}$ are conditionally negative definite. This is a characterisation analogous to Loewner's for operator monotone functions. It is well-known that $f(t)=t^{r}$ is operator convex for $1 \leq r \leq 2$.

The proof of Theorem 1.1 is given in Section 2. We also indicate how the proofs for the parts already given in [4] and [7] can be considerably simplified. The inertia of the matrix $\left[\left(p_{i}+p_{j}\right)^{r}\right]$ has been studied by Bhatia and Jain in [5]. Some ideas in our proofs are similar to the ones used there.

\section{Proofs and Remarks}

Let $X$ be an $n \times n$ nonsingular matrix. The transformation $A \mapsto X^{*} A X$ on Hermitian matrices is called a congruence. The Sylvester Law of Inertia says that

$$
\operatorname{In}\left(X^{*} A X\right)=\operatorname{In} A \text { for all } X \in G L(n) \text {. }
$$


Let $D$ be the diagonal matrix

$$
D=\operatorname{diag}\left(p_{1}, \ldots, p_{n}\right) .
$$

Then for every $r$

$$
L_{-r}=-D^{-r} L_{r} D^{-r}
$$

Hence by Sylvester's Law

$$
\text { In } L_{r}=\left(i_{1}, i_{2}, i_{3}\right) \Leftrightarrow \operatorname{In} L_{-r}=\left(i_{3}, i_{2}, i_{1}\right) .
$$

Thus all statements about In $L_{r}$ for $r>0$ give information about In $L_{-r}$ as well.

Make the substitution $p_{i}=e^{2 x_{i}}, x_{i} \in \mathbb{R}$. A simple calculation shows that

$$
L_{r}=\left[\frac{e^{r x_{i}}}{e^{x_{i}}} \frac{\sinh r\left(x_{i}-x_{j}\right)}{\sinh \left(x_{i}-x_{j}\right)} \frac{e^{r x_{j}}}{e^{x_{j}}}\right] .
$$

In other words,

$$
L_{r}=\Delta \widetilde{L}_{r} \Delta,
$$

where $\Delta=\operatorname{diag}\left(e^{(r-1) x_{1}}, \ldots, e^{(r-1) x_{n}}\right)$, and

$$
\widetilde{L}_{r}=\left[\frac{\sinh r\left(x_{i}-x_{j}\right)}{\sinh \left(x_{i}-x_{j}\right)}\right] .
$$

By Sylvester's Law In $L_{r}=$ In $\widetilde{L}_{r}$. Several properties of $L_{r}$ can be studied via $\widetilde{L}_{r}$, and vice versa. This has been a very effective tool in deriving operator inequalities; see, the work of Bhatia and Parthasarathy [6] and that of Hiai and Kosaki 9, 10, 11, 14.

When $n=2$ we have

$$
\widetilde{L}_{r}=\left[\begin{array}{cc}
r & \frac{\sinh r\left(x_{1}-x_{2}\right)}{\sinh \left(x_{1}-x_{2}\right)} \\
\frac{\sinh r\left(x_{1}-x_{2}\right)}{\sinh \left(x_{1}-x_{2}\right)} & r
\end{array}\right] .
$$

So $\operatorname{det} \widetilde{L}_{r}=r^{2}-\frac{\sinh ^{2} r\left(x_{1}-x_{2}\right)}{\sinh ^{2}\left(x_{1}-x_{2}\right)}$. Thus $\operatorname{det} \widetilde{L}_{r}$ is positive for $0<r<1$, zero for $r=1$, and negative for $r>1$. One eigenvalue of $\widetilde{L}_{r}$ is always positive, and this shows that the second eigenvalue is positive, zero, or negative depending on whether $0<r<1, r=1$, or $r>1$, respectively. This establishes Theorem 1.1 in the simplest case $n=2$.

An interesting corollary can be deduced at this stage. According to the two theorems of Loewner mentioned in Section $1, f$ is operator monotone if and only if all Loewner matrices $L_{f}$ are positive semidefinite, and $f(t)=t^{r}$ is operator monotone if and only if $0 \leq r \leq 1$. Consequently, if $r>1$, then there exists an $n$, and positive numbers $p_{1}, \ldots, p_{n}$ such that the associated Loewner matrix (2) is not positive definite. We can assert more:

Proposition 2.1. Let $r>1$. Then for every $n \geq 2$, and for every choice of $p_{1}, \ldots, p_{n}$, the matrix $L_{r}$ defined in (2) has at least one negative eigenvalue. 
Proof Consider the $2 \times 2$ top left submatrix of $L_{r}$. This is a Loewner matrix. By Theorem 1.1 it has one negative eigenvalue. So, by Cauchy's interlacing principle, the $n \times n$ matrix $L_{r}$ has at least one negative eigenvalue.

The Sylvester Law has a generalisation that is useful for us. Let $n \geq r$, and let $A$ be an $r \times r$ Hermitian matrix and $X$ an $r \times n$ matrix of rank $r$. Then

$$
\text { In } X^{*} A X=\operatorname{In} A+(0, n-r, 0) \text {. }
$$

A proof of this may be found in [5]. This permits a simple transparent proof of Part (ii) of Theorem 1.1. (This part has already been proved in [4.) When $r$ is a positive integer we have

$$
L_{r}=\left[p_{i}^{r-1}+p_{i}^{r-2} p_{j}+\cdots+p_{j}^{r-1}\right]=W^{*} V W,
$$

where $W$ is the $r \times n$ Vandermonde matrix

$$
W=\left[\begin{array}{cccc}
1 & 1 & \cdots & 1 \\
p_{1} & p_{2} & \cdots & p_{n} \\
\cdot & \cdot & \cdots & \cdot \\
p_{1}^{r-1} & p_{2}^{r-1} & \cdots & p_{n}^{r-1}
\end{array}\right]
$$

and $V$ is the $r \times r$ antidiagonal matrix with all entries 1 on its sinister diagonal and all its other entries equal to 0 . If $r=2 k$, the matrix $V$ has $k$ of its eigenvalues equal to 1 , and the other $k$ equal to -1 . If $r=2 k-1$, then $k$ of its eigenvalues are equal to 1 , and $k-1$ are equal to -1 . So, statement (ii) of Theorem 1.1 follows from the generalised Sylvester's Law (13). Next we prove statement (i).

Let $c_{1}, c_{2}, \ldots, c_{n}$ be real numbers, not all of which are zero. Let $f$ be the function on $(0, \infty)$ defined as

$$
f(x)=\sum_{j=1}^{n} c_{j} \frac{x^{r}-p_{j}^{r}}{x-p_{j}} .
$$

Theorem 2.2. Let $r$ be a positive real number not equal to $1,2, \ldots, n-1$. Then the function $f$ defined in (14) has at most $n-1$ zeros in $(0, \infty)$.

Proof Let $r_{1}<r_{2}<\cdots<r_{m}$, and let $a_{1}, \ldots, a_{m}$ be real numbers not all of which are zero. Then the function

$$
g(x)=\sum_{j=1}^{m} a_{j} x^{r_{j}},
$$

has at most $m-1$ zeros in $(0, \infty)$. This is a well-known fact, and can be found in e.g., [16], p.46.

Now let $f$ be the function defined in (14) and let

$$
g(x)=f(x) \prod_{j=1}^{n}\left(x-p_{j}\right) .
$$


Then $g$ can be expressed in the form (15) with $m=2 n$ and

$$
\left\{r_{1}, \ldots, r_{2 n}\right\}=\{0,1, \ldots, n-1, r, r+1, \ldots, r+n-1\} .
$$

Further, we have $g(x)=x^{r} h_{1}(x)-h_{2}(x)$, where

$$
h_{1}(x)=\sum_{i=1}^{n} c_{i} \prod_{j \neq i}\left(x-p_{j}\right), h_{2}(x)=\sum_{i=1}^{n} c_{i} p_{i}^{r} \prod_{j \neq i}\left(x-p_{j}\right) .
$$

Both $h_{1}$ and $h_{2}$ are Lagrange interpolation polynomials of degree at most $n-1$. Since not all $c_{i}$ are zero, neither of these polynomials is identically zero. So, if $r \neq 1,2, \ldots, n-1$, then $g$ is not the zero function.

Hence the function $g$ defined by (16) has at most $2 n-1$ zeros in $(0, \infty)$. Of these, $n$ zeros occur at $x=p_{j}, 1 \leq j \leq n$. So $f$ has at most $n-1$ zeros in $(0, \infty)$.

Corollary 2.3. Let $r$ be a positive real number different from $1,2, \ldots, n-1$. Then the matrix $L_{r}$ defined in (2) is nonsingular.

Proof The matrix $L_{r}$ is singular if and only if there exists a nonzero vector $c=\left(c_{1}, \ldots, c_{n}\right)$ such that $L_{r}(c)=0$. In other words there exist real numbers $c_{1}, \ldots, c_{n}$, not all zero, such that

$$
\sum_{j=1}^{n} c_{j} \frac{p_{i}^{r}-p_{j}^{r}}{p_{i}-p_{j}}=0
$$

for $i=1,2, \ldots, n$. But then the function $f(x)$ in (14) would have $n$ zeros, viz., $x=p_{1}, \ldots, p_{n}$. That is not possible.

We have proved Part (i) of Theorem 1.1. Part (iv) follows from this. If the inertia of $L_{r}$ were to change at some point $r_{0}>n-1$, then one of the eigenvalues has to change sign at $r_{0}$. This is ruled out as $L_{r}$ is nonsingular for all $r>n-1$.

Our argument shows that if $p_{1}<p_{2}<\cdots<p_{n}$ and $q_{1}<q_{2}<\cdots<q_{n}$ are two $n$-tuples of positive real numbers, then the matrix $\left[\frac{p_{i}^{r}-q_{j}^{r}}{p_{i}-q_{j}}\right]$ is nonsingular for every positive $r$ different from $1,2, \ldots, n-1$.

An $n \times n$ real matrix $A$ is said to be strictly sign-regular (SSR for short) if for every $1 \leq k \leq n$, all $k \times k$ sub-determinants of $A$ are nonzero and have the same sign. If this is true for every $1 \leq k \leq r$ for some $r<n$, then we say that $A$ is in the class SSR . Sign-regular matrices and kernels are studied extensively in [15.

We have noted above that if $r$ is any positive real number and $k$ is any positive integer not greater than $r$, then every $k \times k$ matrix of the form $\left[\frac{p_{i}^{r}-q_{j}^{r}}{p_{i}-q_{j}}\right]$ is nonsingular. Let $L_{r}$ be an $n \times n$ Loewner matrix. Let $r \neq 1,2, \ldots, n-1$. Using a homotopy argument one can see that all $k \times k$ sub-determinants of $L_{r}$ are nonzero and have the same sign. Thus $L_{r}$ is an SSR matrix. If $r=1,2, \ldots, n-1$, 
then the same argument shows that for $k \leq r$ all $k \times k$ sub-determinants of $L_{r}$ are nonzero and have the same sign. In other words, $L_{r}$ is an $\mathrm{SSR}_{\mathrm{r}}$ matrix.

Let $A$ be any matrix with eigenvalues $\lambda_{1}, \lambda_{2}, \cdots, \lambda_{n}$ arranged so that $\left|\lambda_{1}\right| \geq$ $\left|\lambda_{2}\right| \geq \cdots \geq\left|\lambda_{n}\right|$. The Perron theorem tells us that if $A$ is entrywise positive, then $\lambda_{1}>0$ and $\lambda_{1}$ is a simple eigenvalue of $A$. (See[13], p. 526). Applying this to successive exterior powers, we see that all eigenvalues of an SSR matrix are simple, and the $r$ nonzero eigenvalues of an $\mathrm{SSR}_{\mathrm{r}}$ matrix of rank $r$ are simple. This proves Part (v) of Theorem 1.1.

We now turn to proving Part (iii). Using the identity

$$
\frac{p_{i}^{r}-p_{j}^{r}}{p_{i}-p_{j}}=\frac{p_{i}^{r-1}\left(p_{i}-p_{j}\right)+p_{i}\left(p_{i}^{r-2}-p_{j}^{r-2}\right) p_{j}+\left(p_{i}-p_{j}\right) p_{j}^{r-1}}{p_{i}-p_{j}}
$$

we see that for every $r \in \mathbb{R}$,

$$
L_{r}=D^{r-1} E+D L_{r-2} D+E D^{r-1},
$$

where $D$ is the diagonal matrix in (8) and $E$ is the $n \times n$ matrix with all its entries equal to one.

By Loewner's Theorem $L_{r}$ is positive definite for $0<r<1$, and because of (10) it is negative definite for $-1<r<0$. Now suppose $1<r<2$. Let $x$ be any nonzero vector in the space $\mathcal{H}_{1}$ defined in $(6)$. Note that this $(n-1)$-dimensional space is the kernel of the matrix $E$. Using (17) we have

$$
\left\langle x, L_{r} x\right\rangle=\left\langle x, D^{r-1} E x\right\rangle+\left\langle x, D L_{r-2} D x\right\rangle+\left\langle x, E D^{r-1} x\right\rangle .
$$

The first and the third term on the right hand side are zero because $E x=0$. So,

$$
\left\langle x, L_{r} x\right\rangle=\left\langle y, L_{r-2} y\right\rangle,
$$

where $y=D x$. The last inner product is negative because $L_{r-2}<0$. Thus $\left\langle x, L_{r} x\right\rangle<0$ for all $x \in \mathcal{H}_{1}$. In other words, $L_{r}$ is conditionally negative definite if $1<r<2$. The same argument shows that $L_{r}$ is conditionally positive definite if $2<r<3$ (because in this case $L_{r-2}$ is positive definite). This was proved in [7] by more elaborate arguments. In particular, we have

$$
\text { In } L_{r}=(1,0, n-1) \text {, if } 1<r<2 \text {, }
$$

and

$$
\text { In } L_{r}=(n-1,0,1) \text {, if } 2<r<3 .
$$

We note here that if $n=3$, then because of Part (iv) already proved we have In $L_{r}=(2,0,1)$ for all $r>2$. So the theorem is completely proved for $n=3$.

Let $n>3$ and suppose $3<r<4$. Now consider the space

$$
\begin{aligned}
\mathcal{H}_{2} & =\left\{x: \sum x_{i}=0, \sum p_{i} x_{i}=0\right\} \\
& =\{x: E x=0, E D x=0\} .
\end{aligned}
$$


This space is of dimension $n-2$, being the orthogonal complement of the span of the vectors $e=(1,1, \ldots 1)$ and $p=\left(p_{1}, p_{2}, \ldots, p_{n}\right)$. Let $x \in \mathcal{H}_{2}$. Again using the relation (17) we see that

$$
\left\langle x, L_{r} x\right\rangle=\left\langle y, L_{r-2} y\right\rangle
$$

where $y=D x$. Since $E D x=0, y$ is in $\mathcal{H}_{1}$, and since $1<r-2<2$, we have $\left\langle x, L_{r} x\right\rangle<0$. This is true for all $x \in \mathcal{H}_{2}$. So, by the minmax principle $L_{r}$ has at least $n-2$ negative eigenvalues. The case $n=3$ of the theorem already proved shows that $L_{r}$ has a $3 \times 3$ principal submatrix with two positive eigenvalues. So, by Cauchy's interlacing principle, $L_{r}$ has at least two positive eigenvalues. Thus $L_{r}$ has exactly two positive and $n-2$ negative eigenvalues. In other words,

$$
\text { In } L_{r}=(2,0, n-2) \text { for } 3<r<4 \text {. }
$$

At this stage note that the Theorem is completely proved for $n=4$. Now let $n>4$, and consider the case $4<r<5$. Arguing as before $\left\langle x, L_{r} x\right\rangle>0$ for all $x \in \mathcal{H}_{2}$. So $L_{r}$ has at least $n-2$ positive eigenvalues. It also has a $4 \times 4$ principal submatrix with two negative eigenvalues. Hence

$$
\text { In } L_{r}=(n-2,0,2) \text { for } 4<r<5 \text {. }
$$

The argument can be continued, introducing the space

$$
\begin{aligned}
\mathcal{H}_{3} & =\left\{x: \sum x_{i}=0, \sum p_{i} x_{i}=0, \sum p_{i}^{2} x_{i}=0\right\} \\
& =\left\{x: E x=0, E D x=0, E D^{2} x=0\right\}
\end{aligned}
$$

at the next stage. Using this we can prove statement (iii) for $5<r<6$ and $6<r<7$. It is clear now how to complete the proof.

All parts of Theorem 1.1 have now been established.

We end this section with a few questions.

1. Let $f(z)$ be the complex function defined as

$$
f(z)=\operatorname{det}\left[\frac{p_{i}^{z}-p_{j}^{z}}{p_{i}-p_{j}}\right] .
$$

Our analysis has shown that $f$ has zeros at $z=0, \pm 1, \pm 2, \ldots, \pm n-1$; these zeros have multiplicities $n, n-1, \ldots, 1$, respectively; and these are the only real zeros of $f$. It might be of interest to find what other zeros $f$ has in the complex plane.

2. When $n=3$, calculations show that

$$
\operatorname{det} L_{3}=-\left(p_{1}-p_{2}\right)^{2}\left(p_{1}-p_{3}\right)^{2}\left(p_{2}-p_{3}\right)^{2}
$$


and

$$
\begin{aligned}
\operatorname{det} L_{4}= & -2\left(p_{1}-p_{2}\right)^{2}\left(p_{1}-p_{3}\right)^{2}\left(p_{2}-p_{3}\right)^{2} \\
& \left\{\left(p_{1}+p_{2}+p_{3}\right)\left(p_{1} p_{2}+p_{1} p_{3}+p_{2} p_{3}\right)+p_{1} p_{2} p_{3}\right\} .
\end{aligned}
$$

It might be of interest to find formulas for the determinants of the matrices $L_{m}$ for integers $m$.

3. Two of the authors have studied the matrix $P_{r}=\left[\left(p_{i}+p_{j}\right)^{r}\right]$ in [5]. It turns out that In $P_{r}=\operatorname{In} L_{r+1}$ for all $r>0$. Why should this be so, and are there other interesting connections between these two matrix families?

Acknowledgements. The work of R. Bhatia is supported by a J. C. Bose National Fellowship, of S. Friedland by the NSF grant DMS-1216393, and of T. Jain by a SERB Women Excellence Award. The authors thank John Holbrook, Roger Horn, Olga Holtz and Lek-Heng Lim for illuminating discussions. The completion of this work was facilitated by the workshop "Positivity, graphical models and modeling of complex multivariate dependencies" at the American Institute of Mathematics in October 2014. The authors thank the organisers and the participants of this workshop.

\section{References}

[1] R. Bhatia, Matrix Analysis, Springer, 1997.

[2] R. Bhatia, Positive Definite Matrices, Princeton Univ. Press, 2007.

[3] R. Bhatia, First and second order perturbation bounds for the operator absolute value, Linear Algebra Appl., 208 (1994) 367-376.

[4] R. Bhatia and J. A. Holbrook, Fréchet derivatives of the power function, Indiana Univ. Math. J., 49(2000) 1155-1173.

[5] R. Bhatia and T. Jain, Inertia of the matrix $\left[\left(p_{i}+p_{j}\right)^{r}\right]$, to appear in J. Spectral Theory.

[6] R. Bhatia and K. R. Parthasarathy, Positive definite functions and operator inequalities, Bull. London Math. Soc., 32 (2000) 214-228.

[7] R. Bhatia and T. Sano, Loewner matrices and operator convexity, Math. Ann., 344 (2009) 703-716.

[8] R. Bhatia and K. B. Sinha, Variation of real powers of positive operators, Indiana Univ. Math. J., 43 (1994) 913-925. 
[9] F. Hiai and H. Kosaki, Comparison of various means for operators, J. Funct. Anal., 163 (1999) 300-323.

[10] F. Hiai and H. Kosaki, Means for matrices and comparison of their norms, Indiana Univ. Math. J., 48 (1999) 899-936.

[11] F. Hiai and H. Kosaki, Means of Hilbert Space Operators, Springer 2003.

[12] R. A. Horn, The Hadamard product. Matrix theory and applications, Proc. Sympos. Appl. Math., 40 (1989) 87-169.

[13] R. A. Horn and C. R. Johnson, Matrix Analysis, Second edition, Cambridge Univ. Press, 2013.

[14] H. Kosaki, Arithmetic-geometric mean and related inequalities for operators, J. Funct. Anal., 156 (1998) 429-451.

[15] S. Karlin, Total Positivity, Stanford University Press, 1968.

[16] G. Pólya and G. Szegö, Problems and Theorems in Analysis, Volume II, 4th ed., Springer, 1971. 